\title{
Multiscale Probability Transformation of Basic Probability Assignment
}

\author{
Meizhu Li, ${ }^{1}$ Xi Lu, ${ }^{1,2}$ Qi Zhang, ${ }^{1}$ and Yong Deng ${ }^{1,3,4}$ \\ ${ }^{1}$ School of Computer and Information Science, Southwest University, Chongqing 400715, China \\ ${ }^{2}$ School of Hanhong, Southwest University, Chongqing 400715, China \\ ${ }^{3}$ School of Automation, Northwestern Polytechnical University, Xian, Shaanxi 710072, China \\ ${ }^{4}$ School of Engineering, Vanderbilt University, Nashville, TN 37235, USA
}

Correspondence should be addressed to Yong Deng; ydeng@swu.edu.cn

Received 16 June 2014; Accepted 2 October 2014; Published 20 October 2014

Academic Editor: Mohamed Abd El Aziz

Copyright (C) 2014 Meizhu Li et al. This is an open access article distributed under the Creative Commons Attribution License, which permits unrestricted use, distribution, and reproduction in any medium, provided the original work is properly cited.

\begin{abstract}
Decision making is still an open issue in the application of Dempster-Shafer evidence theory. A lot of works have been presented for it. In the transferable belief model (TBM), pignistic probabilities based on the basic probability assignments are used for decision making. In this paper, multiscale probability transformation of basic probability assignment based on the belief function and the plausibility function is proposed, which is a generalization of the pignistic probability transformation. In the multiscale probability function, a factor $q$ based on the Tsallis entropy is used to make the multiscale probabilities diversified. An example showing that the multiscale probability transformation is more reasonable in the decision making is given.
\end{abstract}

\section{Introduction}

Since first proposed by Dempster [1] and then developed by Shafer [2], the Dempster-Shafer theory of evidence, which is also called Dempster-Shafer theory or evidence theory, has been paid much attention for a long time and continually attracted growing interests [3-5]. Even as a theory of reasoning under the uncertain environment, DempsterShafer theory has an advantage of directly expressing the "uncertainty" by assigning the probability to the subsets of the set composed of multiple objects rather than to each of the individual objects, so it has been widely used in many fields, such as statistical learning $[6,7]$, classification and clustering [8-11], decision making [12-14], knowledge reasoning [15, 16], risk assessment and evaluation [17, 18], and others [19-21].

To improve the Dempster-Shafer theory of evidence, many studies have been devoted for combination rule of evidence, confliction problem, generation of mass function $[22,23]$, uncertain measure of evidence $[24,25]$, and so on [26-28]. The basic rule of combination is Dempster's rule of combination (orthogonal sum). It needs a normalization step in order to preserve the basic properties of the belief functions. In [29], Zadeh has underlined that this normalization involves counterintuitive behaviours. In order to solve the problem of conflict management, Yager [30], Smets [31], and Murphy [32] and more recently Liu [33] and Lefèvre and Elouedi [34] have proposed other combination rules.

One open issue of evidence theory is the decision making based on the basic probability assignments, and many works have been done to construct a reasonable model for the decision making. In the transferable belief model (TBM) [35], pignistic probabilities are used for decision making [36]. The transferable belief model is presented to represent quantified beliefs based on belief functions. TBM was constructed by two levels: the credal level where beliefs are entertained and quantified by belief functions and the pignistic level where beliefs can be used to make decisions and are quantified by probability functions. The main idea of the pignistic probability transformation is to transform the multielement subsets into singleton subsets by an average method. Because in some examples the pignistic transformation method produces results that appear to be inconsistent with Dempster's rule of combination, Cobb and Shenoy [37] proposed a new plausibility transformation method for translating 
Dempster-Shafer (D-S) belief function models to probability models. Other works have been done to obtain a more reasonable transformation method [38-40].

Among the previous research, the pignistic probability transformation is the most widely used, but it is not reasonable enough to describe the unknown for the multielement subsets. Hence, a generalization of the pignistic probability transformation called multiscale probability transformation of basic probability assignment is proposed in this paper, which is based on the belief function and the plausibility function. The proposed function can be calculated with the difference between the belief function and the plausibility function; we call it multiscale probability function and denote it as a function MulP. In the multiscale probability function, a factor $q$ based on the Tsallis entropy [41] is used to make the multiscale probabilities diversified. When the value of $q$ equals 0 , the proposed multiscale probability transformation can be degenerated as the pignistic probability transformation.

The rest of this paper is organized as follows. Section 2 introduces some basic preliminaries about the DempsterShafer theory and the pignistic probability transformation. In Section 3, the multiscale probability transformation is presented. Section 4 uses an example to illustrate the effectiveness of the multiscale probability transformation. Conclusion is given in Section 5 .

\section{Preliminaries}

2.1. Dempster-Shafer Theory of Evidence. Dempster-Shafer theory of evidence $[1,2]$, also called Dempster-Shafer theory or evidence theory, is used to deal with uncertain information. As an effective theory of evidential reasoning, Dempster-Shafer theory has an advantage of directly expressing various uncertainties. This theory needs weaker conditions than Bayesian theory of probability, so it is often regarded as an extension of the Bayesian theory. For completeness of the explanation, a few basic concepts are introduced as follows.

Definition 1. Let $\Omega$ be a mutually exclusive and collectively exhaustive set, which is indicted by:

$$
\Omega=\left\{E_{1}, E_{2}, \ldots, E_{i}, \ldots, E_{N}\right\}
$$

The set $\Omega$ is called frame of discernment. The power set of $\Omega$ is indicated by $2^{\Omega}$, where

$$
\begin{gathered}
2^{\Omega}=\left\{\emptyset,\left\{E_{1}\right\}, \ldots,\left\{E_{N}\right\},\left\{E_{1}, E_{2}\right\}, \ldots,\right. \\
\left.\left\{E_{1}, E_{2}, \ldots, E_{i}\right\}, \ldots, \Omega\right\} .
\end{gathered}
$$

If $A \in 2^{\Omega}, A$ is called a proposition.

Definition 2. For a frame of discernment $\Omega$, a mass function is a mapping $m$ from $2^{\Omega}$ to $[0,1]$, formally defined by

$$
m: 2^{\Omega} \longrightarrow[0,1]
$$

which satisfies the following condition:

$$
m(\emptyset)=0, \quad \sum_{A \in 2^{\Omega}} m(A)=1 .
$$

In Dempster-Shafer theory, a mass function is also called a basic probability assignment (BPA). If $m(A)>0, A$ is called a focal element, and the union of all focal elements is called the core of the mass function.

Definition 3. For a proposition $A \subseteq \Omega$, the belief function Bel : $2^{\Omega} \rightarrow[0,1]$ is defined as

$$
\operatorname{Bel}(A)=\sum_{B \subseteq A} m(B) .
$$

The plausibility function $\mathrm{Pl}: 2^{\Omega} \rightarrow[0,1]$ is defined as

$$
\operatorname{Pl}(A)=1-\operatorname{Bel}(\bar{A})=\sum_{B \cap A \neq \emptyset} m(B),
$$

where $\bar{A}=\Omega-A$.

Obviously, $\operatorname{Pl}(A) \geq \operatorname{Bel}(A)$, and these functions Bel and $\mathrm{Pl}$ are the lower limit function and upper limit function of proposition $A$, respectively.

2.2. Pignistic Probability Transformation. In the transferable belief model (TBM) [35], pignistic probabilities are used for decision making. The definition of the pignistic probability transformation is shown as follows.

Definition 4. Let $m$ be a BPA on the frame of discernment $\Omega$. Its associated pignistic probability function $\operatorname{Bet} P_{m}: \Omega \rightarrow$ $[0,1]$ is defined as

$$
\operatorname{Bet} P_{m}(\omega)=\sum_{A \subseteq P(\Omega), \omega \in A} \frac{1}{|A|} \frac{m(A)}{1-m(\phi)}, \quad m(\phi) \neq 1,
$$

where $|W|$ is the cardinality of subset $A$. The process of pignistic probability transformation (PPT) is that basic probability assignment transferred to probability distribution. Therefore, the pignistic betting distance can be easily obtained by PPT.

\section{Multiscale Probability Transformation of Basic Probability Assignment}

In the transferable belief model (TBM) [35], pignistic probabilities are used for decision making. The transferable belief model is presented to represent quantified beliefs based on belief functions. The main idea of the pignistic probability transformation is to transform the multielement subsets into singleton subsets by an average method. Though the pignistic probability transformation is widely used, it is not reasonable in Example 5.

Example 5. Suppose there is a frame of discernment of $a, b$, and $c$; then, the BPA is given as follows:

$$
\begin{gathered}
m(\{a\})=0.2, \quad m(\{b\})=0.7, \\
m(\{b, c\})=0.05, \quad m(\{a, b, c\})=0.05 .
\end{gathered}
$$


In the pignistic probability transformation, for $m(\{a, b, c\})=0.05$, the result will be $a=b=c=0.05 / 3$. Actually, it is not reasonable, as $m(\{a, b, c\})=0.05$ means that the sensor cannot judge to which classes the target belongs, since it represents a meaning of "unknown." In other words, only according to $m(\{a, b, c\})=0.05$, nothing can be obtained except "unknown." In this situation, average is used in the pignistic probability transformation, which is one of the methods to solve the problem. Compared with the average, weighted average is more reasonable in many situations.

In the Dempster-Shafer theory, the plausibility function represents the optimistic estimate of one proposition, and the belief function represents the pessimistic estimate of one proposition. The distance between the plausibility function and the belief function refers to the belief level of one proposition. For one proposition, the bigger the belief level, the bigger the probability. In this paper, the weighted average is represented by the distance between the belief function and the plausibility function, whose definition is shown as follows.

Definition 6. Let $m$ be a BPA on the frame of discernment $\Omega$. The difference function $d_{m}$ is defined as

$$
d_{m}(\omega)=\operatorname{Pl}(\omega)-\operatorname{Bel}(\omega), \quad \omega \in \Omega .
$$

Definition 7. The weight is defined as

$$
\text { Weight }_{m}(\omega)=\frac{d_{m}(\omega)}{\sum_{\alpha \in A}^{|A|} d_{m}(\alpha)}, \quad \omega \in A, A \subseteq P(\Omega) .
$$

Based on the weighted average idea, a factor $q$, which is proposed in the Tsallis entropy [41], is used to highlight the weights. Thus, the definition of multiscale probability function MulP is shown as follows.

Definition 8. Let $m$ be a BPA on the frame of discernment $\Omega$. Its associated multiscale probability function $\operatorname{Mul}_{m}: \Omega \rightarrow$ $[0,1]$ on $\Omega$ is defined as

$$
\begin{array}{r}
\operatorname{Mul} P_{m}(\omega) \\
=\sum_{A \subseteq P(\Omega), \omega \in A}\left(\frac{(\operatorname{Pl}(\omega)-\operatorname{Bel}(\omega))^{q}}{\sum_{\alpha \in A}^{|A|}(\operatorname{Pl}(\alpha)-\operatorname{Bel}(\alpha))^{q}} \frac{m(A)}{1-m(\phi)}\right), \\
m(\phi) \neq 1,
\end{array}
$$

where $|W|$ is the cardinality of subset $A . q$ is a factor based on the Tsallis entropy to amend the proportion of the interval. The transformation between $m$ and $\operatorname{Mul}_{m}$ is called the multiscale probability transformation.

Actually, the part of (11) $(\operatorname{Pl}(\omega)-\operatorname{Bel}(\omega))^{q} / \sum_{\alpha \in W}^{|W|}$ $(\operatorname{Pl}(\alpha)-\operatorname{Bel}(\alpha))^{q}$ denotes the weight of element $\omega$ based on normalization, which is replaced by the averaged $1 /|W|$ in the pignistic probability function.

Theorem 9. Let $m$ be a BPA on the frame of discernment $\Omega$. Its associated multiscale probability $\mathrm{Mul}_{m}$ on $\Omega$ is degenerated as the pignistic probability $\operatorname{Bet} P_{m}$ when q equals 0 .
Proof. When $q$ equals 0 and $(\operatorname{Pl}(\omega)-\operatorname{Bel}(\omega))^{q}$ equals 1 , the multiscale probability function will be calculated as follows:

$$
\begin{aligned}
& \operatorname{Mul}_{m}(\omega) \\
& =\sum_{A \subseteq \Omega, \omega \in A}\left(\frac{1}{\sum_{\alpha \in A}^{|A|} 1} \cdot \frac{m^{\Omega}(W)}{\left(1-m^{\Omega}(\phi)\right)}\right), \quad \forall \omega \in \Omega .
\end{aligned}
$$

Then, it can obtain

$$
\begin{aligned}
& \operatorname{Mul}_{m}(\omega) \\
& =\sum_{A \subseteq \Omega, \omega \in A}\left(\frac{1}{|A|} \cdot \frac{m^{\Omega}(A)}{\left(1-m^{\Omega}(\phi)\right)}\right), \quad \forall \omega \in \Omega .
\end{aligned}
$$

From (12) and (13), we can see that when the value of $q$ equals 0 , the proposed multiscale probability function can be degenerated as the pignistic probability function.

Theorem 10. Let $m$ be a BPA on the frame of discernment $\Omega$. If the belieffunction equals the plausibility function, its associated multiscale probability $\mathrm{Mul}_{m}$ is degenerated as the pignistic probability Bet $P_{m}$.

Proof. Given a BPA $m$ on the frame of discernment $\Omega$, for each $\omega \in \Omega$, when the belief function equals the plausibility function, namely, $\operatorname{Bel}(\omega)=\operatorname{Pl}(\omega)$, and the bel is a probability distribution $P$ [35], then $\mathrm{Mul} P$ is equal to $\operatorname{Bet} P$.

For example, let $\Omega$ be a frame of discernment and $\Omega=$ $\{a, b, c\}$; if it is satisfied with $\operatorname{Bel}(a)=\operatorname{Pl}(a), \operatorname{Bel}(b)=\operatorname{Pl}(b)$, and $\operatorname{Bel}(c)=\operatorname{Pl}(c)$, the BPA on the frame must be satisfied with $m(a)+m(b)+m(c)=1$. In this situation, the multiscale probability will be degenerated as the pignistic probability.

Corollary 11. If bel is a probability distribution $P$, then $\mathrm{Mul} P$ is equal to $P$.

Theorem 12. Let $m$ be a BPA on the frame of discernment $\Omega=a, b, c$. If the distances between the belief function and the plausibility function are the same, the multiscale probability transformation can be degenerated as the pignistic probability transformation.

Proof. It is the same as the proof of Theorem 9.

An illustrative example is given to show the calculation of the multiscale probability transformation step by step.

Example 13. Let $\Omega$ be a frame of discernment with 3 elements. We use $a, b$, and $c$ to denote element 1 , element 2 , and element 3 in the frame. One body of BPA is given as follows:

$$
\begin{gathered}
m(\{a\})=0.2, \quad m(\{b\})=0.3, \quad m(\{c\})=0.1, \\
m(\{a, b\})=0.1, \quad m(\{a, b, c\})=0.3 .
\end{gathered}
$$


Step 1. Based on (5) and (6), the values of the belief function and the plausibility function of elements $a, b$, and $c$ can be obtained as follows:

$$
\begin{array}{ll}
\operatorname{Bel}(a)=0.2, & \operatorname{Pl}(a)=0.6, \\
\operatorname{Bel}(b)=0.3, & \operatorname{Pl}(b)=0.7, \\
\operatorname{Bel}(c)=0.1, & \operatorname{Pl}(c)=0.4 .
\end{array}
$$

Step 2. Calculate the distance between the belief function and the plausibility function:

$$
\begin{aligned}
& d_{m}(a)=\operatorname{Pl}(a)-\operatorname{Bel}(a)=0.6-0.2=0.4, \\
& d_{m}(b)=\operatorname{Pl}(b)-\operatorname{Bel}(b)=0.7-0.3=0.4, \\
& d_{m}(c)=\operatorname{Pl}(c)-\operatorname{Bel}(c)=0.4-0.1=0.3 .
\end{aligned}
$$

Step 3. Calculate the weight of each element in $\Omega$. Assume that the value of $q$ equals 1 .

When $A=\{a, b\}$,

$$
\begin{aligned}
\text { Weight }_{m}(a) & =\frac{(\operatorname{Pl}(a)-\operatorname{Bel}(a))}{\sum_{\alpha \in W}^{|W|}(\operatorname{Pl}(\alpha)-\operatorname{Bel}(\alpha))} \\
& =\frac{0.4}{(0.4+0.4)}=0.5,
\end{aligned}
$$$$
\text { Weight }_{m}(b)=\frac{(\operatorname{Pl}(b)-\operatorname{Bel}(b))}{\sum_{\alpha \in W}^{|W|}(\operatorname{Pl}(\alpha)-\operatorname{Bel}(\alpha))}=\frac{0.4}{(0.4+0.4)}=0.5 \text {. }
$$

When $W=\{a, b, c\}$,

$$
\begin{aligned}
\text { Weight }_{m}(a) & =\frac{(\mathrm{Pl}(a)-\operatorname{Bel}(a))}{\sum_{\alpha \in W}^{|W|}(\mathrm{Pl}(\alpha)-\operatorname{Bel}(\alpha))} \\
& =\frac{0.4}{(0.4+0.4+0.3)}=0.364, \\
\text { Weight }_{m}(b) & =\frac{(\mathrm{Pl}(b)-\operatorname{Bel}(b))}{\sum_{\alpha \in W}^{|W|}(\mathrm{Pl}(\alpha)-\operatorname{Bel}(\alpha))} \\
& =\frac{0.4}{(0.4+0.4+0.3)}=0.364, \\
\text { Weight }_{m}(c) & =\frac{(\operatorname{Pl}(c)-\operatorname{Bel}(c))}{\sum_{\alpha \in W}^{|W|}(\mathrm{Pl}(\alpha)-\operatorname{Bel}(\alpha))} \\
& =\frac{0.3}{(0.4+0.4+0.3)}=0.272 .
\end{aligned}
$$

Step 4. The value of the multiscale probability function can be obtained based on the above steps:

$$
\begin{aligned}
& \operatorname{Mul}_{m}(a)=0.2+0.1 * 0.5+0.3 * 0.364=0.3592, \\
& \operatorname{Mul}_{m}(b)=0.3+0.1 * 0.5+0.3 * 0.364=0.4592, \\
& \operatorname{Mul}_{m}(c)=0.1+0.3 * 0.272=0.1816 .
\end{aligned}
$$

\section{Case Study}

In this section, an illustrative example is given to show the effect of the multiscale probability function when the value of $q$ changes.

Example 14. Let $\Omega$ be a frame of discernment with 3 elements; namely, $\Omega=\{a, b, c\}$.

We are given one body of BPAs as follows:

$$
\begin{gathered}
m(\{a\})=0.3, \quad m(\{b\})=0.1, \quad m(\{a, b\})=0.1, \\
m(\{a, c\})=0.2, \quad m(\{a, b, c\})=0.3 .
\end{gathered}
$$

Based on the pignistic probability transformation, the results of the pignistic probability function are shown as follows:

$$
\begin{gathered}
\operatorname{Bet} P_{m}(a)=0.55, \quad \operatorname{Bet} P_{m}(b)=0.25, \\
\operatorname{Bet} P_{m}(c)=0.20 .
\end{gathered}
$$

According to the proposed function in this paper, the results of the multiscale probability function can be obtained through the following steps.

Firstly, the values of belief function and the plausibility function can be obtained as follows:

$$
\begin{array}{cc}
\operatorname{Bel}(a)=0.3, & \operatorname{Pl}(a)=0.9, \\
\operatorname{Bel}(b)=0.1, & \operatorname{Pl}(b)=0.5, \\
\operatorname{Bel}(c)=0, & \operatorname{Pl}(c)=0.5 .
\end{array}
$$

Then, the distances between the belief functions and the plausibility functions can be calculated:

$$
\begin{gathered}
d_{m}(a)=\operatorname{Pl}(a)-\operatorname{Bel}(a)=0.9-0.3=0.6, \\
d_{m}(b)=\operatorname{Pl}(b)-\operatorname{Bel}(b)=0.5-0.1=0.4, \\
d_{m}(c)=\operatorname{Pl}(c)-\operatorname{Bel}(c)=0.5-0=0.5 .
\end{gathered}
$$

Based on the definition of the multiscale probability transformation, the values of $\mathrm{Mul}_{m}$ can be obtained. There are 20 cases where the values of $q$ are starting from Case 1 with $q=0$ and ending with Case 11 when $q=10$ as shown in Table 1 . The values of $\mathrm{Mul} P_{m}$ for these 20 cases are detailed in Table 1 and graphically illustrated in Figure 1.

According to Table 1 and Figure 1, on one hand, when the value of $q$ increased, the probability of the element which has larger weight is increased, and the probability of the element which has smaller weight is decreased. For example, the element $a$ is starting with probability 0.5500 and ending with probability 0.8250 . The element $b$ is starting with probability 0.2500 and ending with probability 0.1061 .

On the other hand, according to Table 1, the option ranking of the values of $\mathrm{Mul}_{m}$ can be obtained. It is starting with $\operatorname{Mul} P_{m}(a)>\operatorname{Mul}_{m}(b)>\operatorname{Mul} P_{m}(c)$ and ending with $\operatorname{Mul} P_{m}(a)>\operatorname{Mul} P_{m}(c)>\operatorname{Mul} P_{m}(b)$. It is mainly because $\operatorname{Mul}_{m}$ is impact of the values of $q$. This principle makes the multiscale probability function have the ability to highlight the proportion of each element in the frame of discernment. 
TABLE 1: The values of multiscale probability function when the values of $q$ change.

\begin{tabular}{lccc}
\hline Cases & $\operatorname{Mul} P(a)$ & $\operatorname{Mul} P(b)$ & $\operatorname{Mul} P(c)$ \\
\hline$q=0$ & 0.5500 & 0.2500 & 0.2000 \\
$q=1$ & 0.5891 & 0.2200 & 0.1909 \\
$q=2$ & 0.6275 & 0.1931 & 0.1794 \\
$q=3$ & 0.6638 & 0.1703 & 0.1659 \\
$q=4$ & 0.5970 & 0.1518 & 0.1512 \\
$q=5$ & 0.7267 & 0.1374 & 0.1360 \\
$q=6$ & 0.7526 & 0.1266 & 0.1208 \\
$q=7$ & 0.7751 & 0.1187 & 0.1062 \\
$q=8$ & 0.7944 & 0.1130 & 0.0926 \\
$q=9$ & 0.8109 & 0.1089 & 0.0801 \\
$q=10$ & 0.8250 & 0.1061 & 0.0689 \\
\hline
\end{tabular}

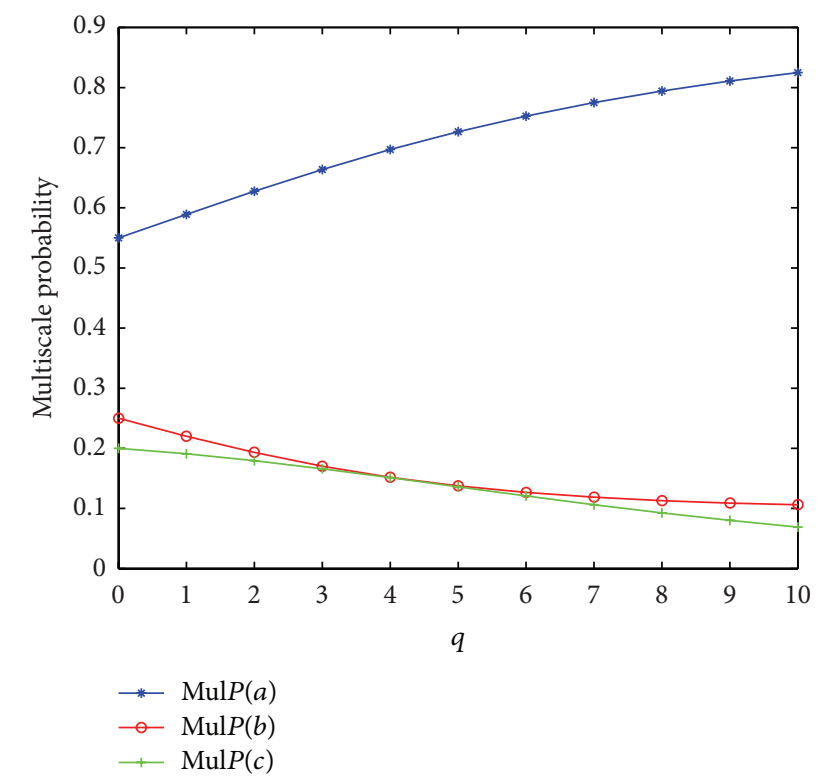

FIGURE 1: The values of multiscale probability function when the values of $q$ change.

Note that when the value of $q$ equals 0 , the values of pignistic probability $\operatorname{Bet} P_{m}$ are the same as the values of multiscale probability $\mathrm{Mul} P_{m}$, which is proposed in this paper. In other words, the multiscale probability function is a generalization of the pignistic probability function.

\section{Conclusion}

In the transferable belief model (TBM), pignistic probabilities are used for decision making. In this paper, a multiscale probability transformation of basic probability assignment based on the belief function and the plausibility function, which is a generalization of the pignistic probability transformation, is proposed. In the multiscale probability function, a factor $q$ is proposed to make the multiscale probability function have the ability to highlight the proportion of each element in the frame of discernment. When the value of $q$ equals 0 , the multiscale probability transformation can be degenerated as the pignistic probability transformation. An illustrative case is provided to demonstrate the effectiveness of the multiscale probability transformation.

\section{Conflict of Interests}

The authors declare that there is no conflict of interests regarding the publication of this paper.

\section{Acknowledgments}

The authors thank the anonymous reviewers for their valuable comments and suggestions that improved this paper. The work is partially supported by the National Natural Science Foundation of China (Grant no. 61174022), Specialized Research Fund for the Doctoral Program of Higher Education (Grant no. 20131102130002), R\&D Program of China (2012BAH07B01), National High Technology Research and Development Program of China (863 Program) (Grant no. 2013AA013801), Open Funding Project of State Key Laboratory of Virtual Reality Technology and Systems, Beihang University (Grant no. BUAA-VR-14KF-02), and Fundamental Research Funds for the Central Universities no. XDJK2015D009.

\section{References}

[1] A. P. Dempster, "Upper and lower probabilities induced by a multivalued mapping," The Annals of Mathematical Statistics, vol. 38, no. 2, pp. 325-339, 1967.

[2] G. Shafer, A Mathematical Theory of Evidence, vol. 1, Princeton University Press, Princeton, NJ, USA, 1976.

[3] R. P. Srivastava and L. Liu, "Applications of belief functions in business decisions: a review," Information Systems Frontiers, vol. 5, no. 4, pp. 359-378, 2003.

[4] T. Denœux, "Maximum likelihood estimation from fuzzy data using the EM algorithm," Fuzzy Sets and Systems, vol. 183, no. 1, pp. 72-91, 2011.

[5] D. Wei, X. Deng, X. Zhang, Y. Deng, and S. Mahadevan, "Identifying influential nodes in weighted networks based on evidence theory," Physica A: Statistical Mechanics and Its Applications, vol. 392, no. 10, pp. 2564-2575, 2013.

[6] F. Cuzzolin, "A geometric approach to the theory of evidence," IEEE Transactions on Systems, Man and Cybernetics Part C: Applications and Reviews, vol. 38, no. 4, pp. 522-534, 2008.

[7] Y. Yang, D. Han, C. Han, and F. Cao, "A novel approximation of basic probability assignment based on rank-level fusion," Chinese Journal of Aeronautics, vol. 26, no. 4, pp. 993-999, 2013.

[8] M.-H. Masson and T. Denœux, "ECM: an evidential version of the fuzzy c-means algorithm," Pattern Recognition, vol. 41, no. 4, pp. 1384-1397, 2008.

[9] Z.-G. Liu, Q. Pan, and J. Dezert, "Evidential classifier for imprecise data based on belief functions," Knowledge-Based Systems, vol. 52, pp. 246-257, 2013.

[10] L. Livi, H. Tahayori, A. Sadeghian, and A. Rizzi, "Distinguishability of interval type-2 fuzzy sets data by analyzing upper and lower membership functions," Applied Soft Computing Journal, vol. 17, pp. 79-89, 2014. 
[11] S. Agrawal, R. Panda, and L. Dora, "A study on fuzzy clustering for magnetic resonance brain image segmentation using soft computing approaches," Applied Soft Computing, vol. 24, pp. 522-533, 2014.

[12] J. Liu, Y. Li, R. Sadiq, and Y. Deng, "Quantifying influence of weather indices on pm based on relation map," Stochastic Environmental Research and Risk Assessment, vol. 28, no. 6, pp. 1323-1331, 2014.

[13] S. Huang, X. Su, Y. Hu, S. Mahadevan, and Y. Deng, "A new decision-making method by incomplete preferences based on evidence distance," Knowledge-Based Systems, vol. 56, pp. 264272, 2014.

[14] C. S. Lin, C. T. Chen, F. S. Chen, and W. Z. Hung, "A novel multiperson game approach for linguistic multicriteria decision making problems," Mathematical Problems in Engineering, vol. 2014, Article ID 592326, 20 pages, 2014.

[15] B. Kang, Y. Deng, R. Sadiq, and S. Mahadevan, "Evidential cognitive maps," Knowledge-Based Systems, vol. 35, pp. 77-86, 2012.

[16] T. Denoeux, "Maximum likelihood estimation from uncertain data in the belief function framework," IEEE Transactions on Knowledge and Data Engineering, vol. 25, no. 1, pp. 119-130, 2013.

[17] X. Zhang, Y. Deng, F. T. S. Chan, P. Xu, S. Mahadevan, and Y. $\mathrm{Hu}$, "IFSJSP: a novel methodology for the Job-Shop Scheduling Problem based on intuitionistic fuzzy sets," International Journal of Production Research, vol. 51, no. 17, pp. 5100-5119, 2013.

[18] X. Deng, Y. Hu, Y. Deng, and S. Mahadevan, "Supplier selection using AHP methodology extended by D numbers," Expert Systems with Applications, vol. 41, no. 1, pp. 156-167, 2014.

[19] S. Chen, Y. Deng, and J. Wu, "Fuzzy sensor fusion based on evidence theory and its application," Applied Artificial Intelligence, vol. 27, no. 3, pp. 235-248, 2013.

[20] X. Deng, Y. Hu, Y. Deng, and S. Mahadevan, "Environmental impact assessment based on D numbers," Expert Systems with Applications, vol. 41, no. 2, pp. 635-643, 2014.

[21] C. Zhang, Y. Hu, F. T. Chan, R. Sadiq, and Y. Deng, "A new method to determine basic probability assignment using core samples," Knowledge-Based Systems, vol. 69, pp. 140-149, 2014.

[22] T. Burger and S. Destercke, "How to randomly generate mass functions," International Journal of Uncertainty, Fuzziness and Knowlege-Based Systems, vol. 21, no. 5, pp. 645-673, 2013.

[23] Z.-G. Liu, Q. Pan, and J. Dezert, "A belief classification rule for imprecise data," Applied Intelligence, vol. 40, no. 2, pp. 214-228, 2014.

[24] M. E. Basiri, A. R. Naghsh-Nilchi, and N. Ghasem-Aghaee, "Sentiment prediction based on dempster-shafer theory of evidence," Mathematical Problems in Engineering, vol. 2014, Article ID 361201, 13 pages, 2014.

[25] X. Deng, X. Lu, F. T. Chan, R. Sadiq, S. Mahadevan, and Y. Deng, "D-CFPR: D numbers extended consistent fuzzy preference relations," Knowledge-Based Systems, 2014.

[26] F. Karahan and S. Ozkan, "On the persistence of income shocks over the life cycle: evidence, theory, and implications," Review of Economic Dynamics, vol. 16, no. 3, pp. 452-476, 2013.

[27] Z. Zhang, C. Jiang, X. Han, D. Hu, and S. Yu, "A response surface approach for structural reliability analysis using evidence theory," Advances in Engineering Software, vol. 69, pp. 37-45, 2014.

[28] X. Zhang, Y. Deng, F. T. Chan, A. Adamatzky, and S. Mahadevan, "Supplier selection based on evidence theory and analytic network process," Proceedings of the Institution of Mechanical Engineers, Part B: Journal of Engineering Manufacture, 2015.
[29] L. A. Zadeh, "A simple view of the dempster-shafer theory of evidence and its implication for the rule of combination," The AI Magazine, vol. 7, no. 2, pp. 85-90, 1986.

[30] R. R. Yager, "On the dempster-shafer framework and new combination rules," Information Sciences, vol. 41, no. 2, pp. 93137, 1987.

[31] P. Smets, "Combination of evidence in the transferable belief model," IEEE Transactions on Pattern Analysis and Machine Intelligence, vol. 12, no. 5, pp. 447-458, 1990.

[32] C. K. Murphy, "Combining belief functions when evidence conflicts," Decision Support Systems, vol. 29, no. 1, pp. 1-9, 2000.

[33] W. Liu, "Analyzing the degree of conflict among belief functions," Artificial Intelligence, vol. 170, no. 11, pp. 909-924, 2006.

[34] E. Lefèvre and Z. Elouedi, "How to preserve the conflict as an alarm in the combination of belief functions?" Decision Support Systems, vol. 56, no. 1, pp. 326-333, 2013.

[35] P. Smets and R. Kennes, "The transferable belief model," Artificial Intelligence, vol. 66, no. 2, pp. 191-234, 1994.

[36] P. Smets, "Decision making in the TBM: the necessity of the pignistic transformation," International Journal of Approximate Reasoning, vol. 38, no. 2, pp. 133-147, 2005.

[37] B. R. Cobb and P. P. Shenoy, "On the plausibility transformation method for translating belief function models to probability models," International Journal of Approximate Reasoning, vol. 41, no. 3, pp. 314-330, 2006.

[38] M. Daniel, "On transformations of belief functions to probabilities," International Journal of Intelligent Systems, vol. 21, no. 3, pp. 261-282, 2006.

[39] J. M. Merigó and M. Casanovas, "Decision making with Dempster-Shafer theory using fuzzy induced aggregation operators," in Recent Developments in the Ordered Weighted Averaging Operators: Theory and Practice, vol. 265 of Studies in Fuzziness and Soft Computing, pp. 209-228, Springer, 2011.

[40] E. Nusrat and K. Yamada, "A descriptive decision-making model under uncertainty: combination of dempster-sha fer theory and prospect theory," International Journal of Uncertainty, Fuzziness and Knowlege-Based Systems, vol. 21, no. 1, pp. 79-102, 2013.

[41] C. Tsallis, "Possible generalization of Boltzmann-Gibbs statistics," Journal of Statistical Physics, vol. 52, no. 1-2, pp. 479-487, 1988. 


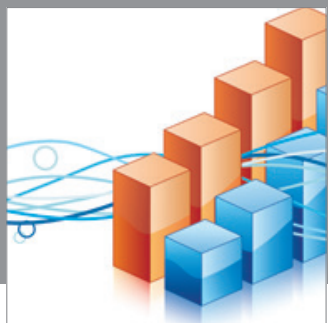

Advances in

Operations Research

mansans

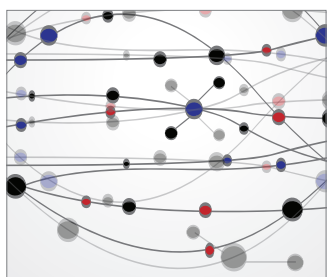

The Scientific World Journal
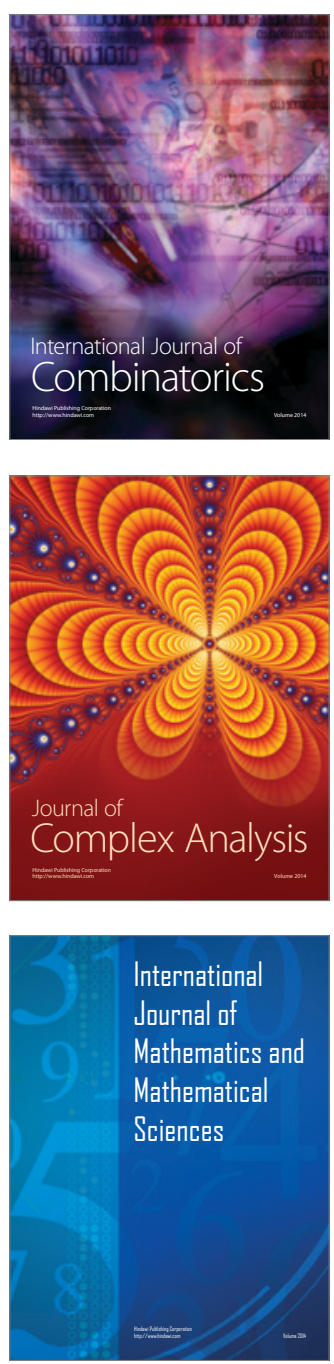
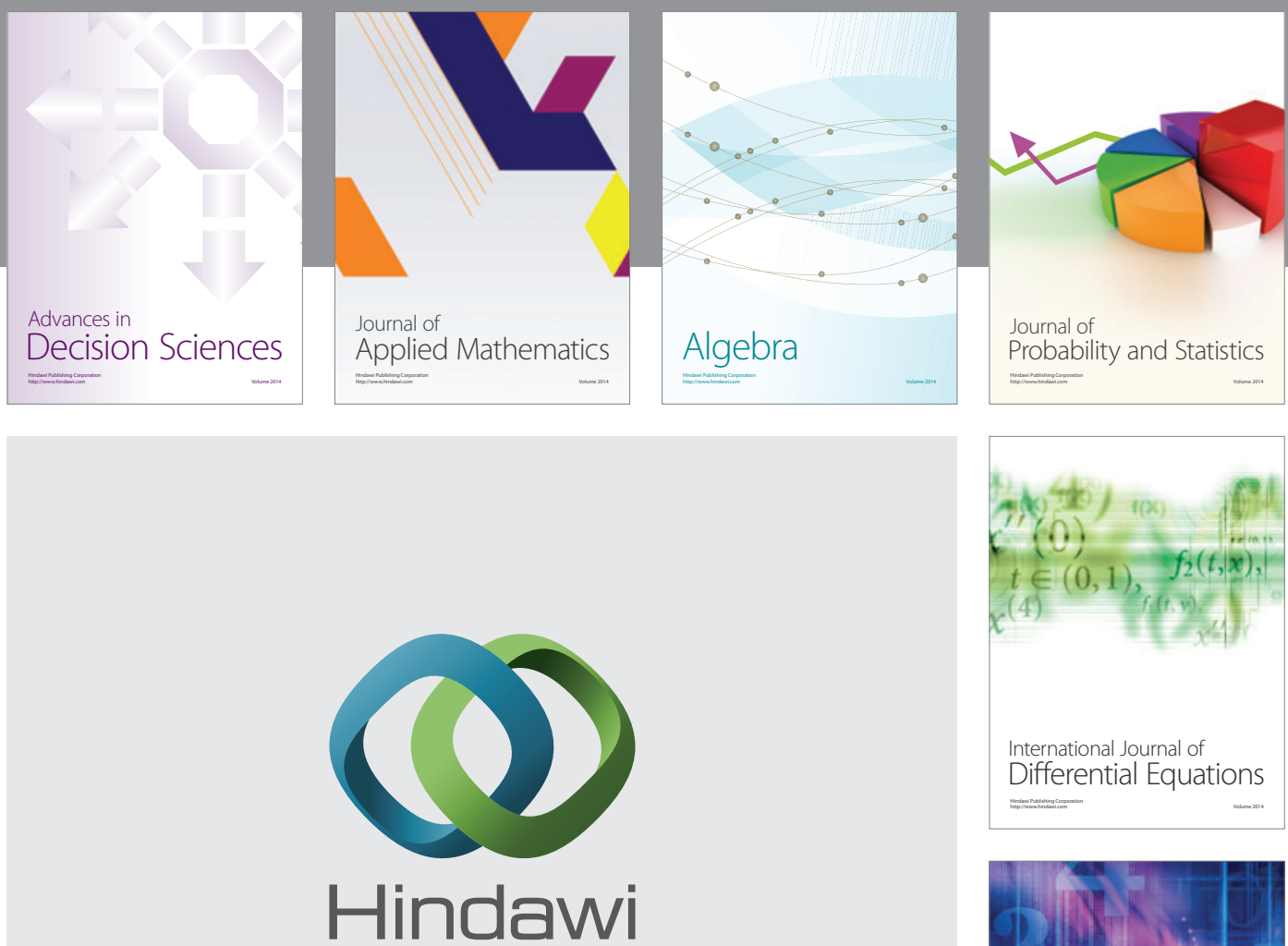

Submit your manuscripts at http://www.hindawi.com
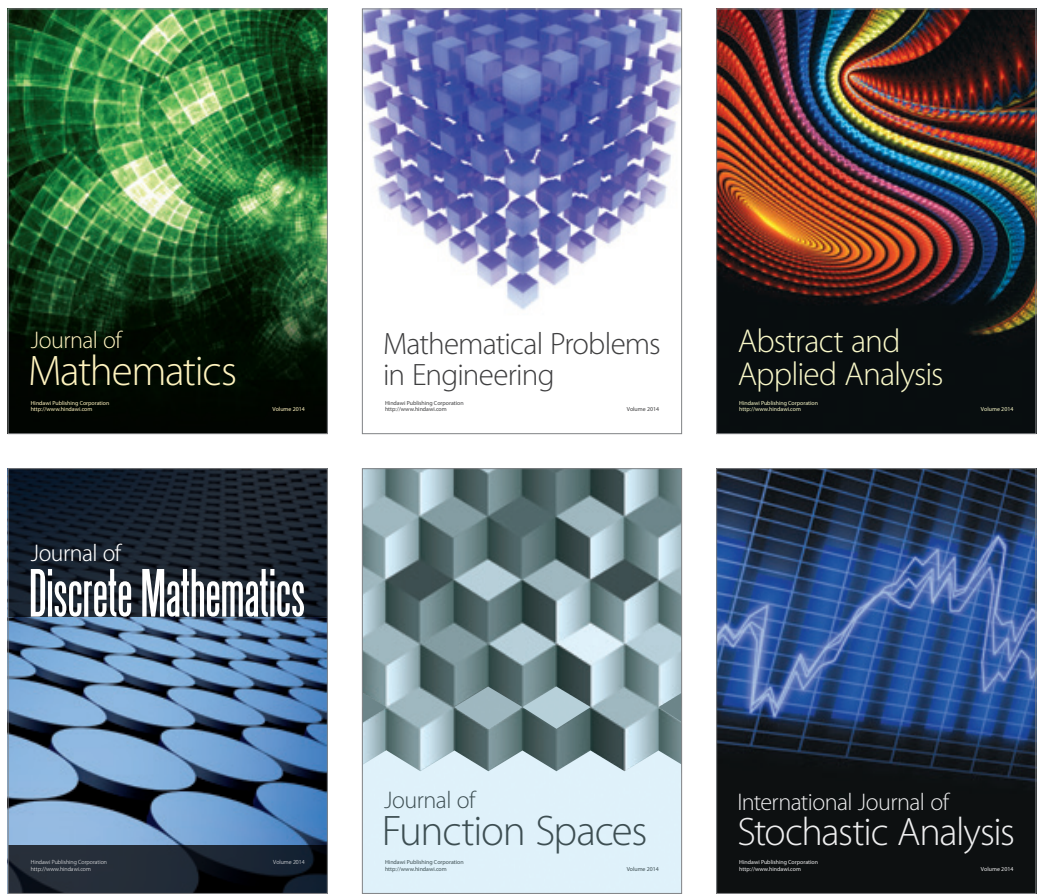

Journal of

Function Spaces

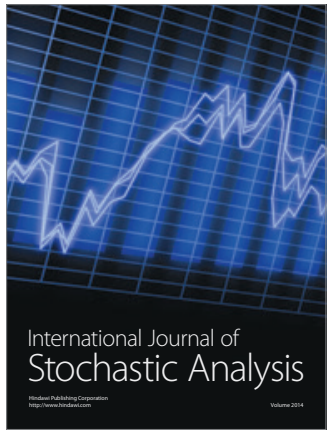

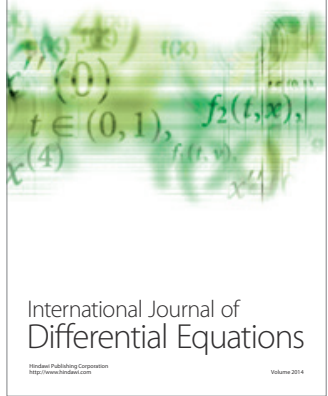
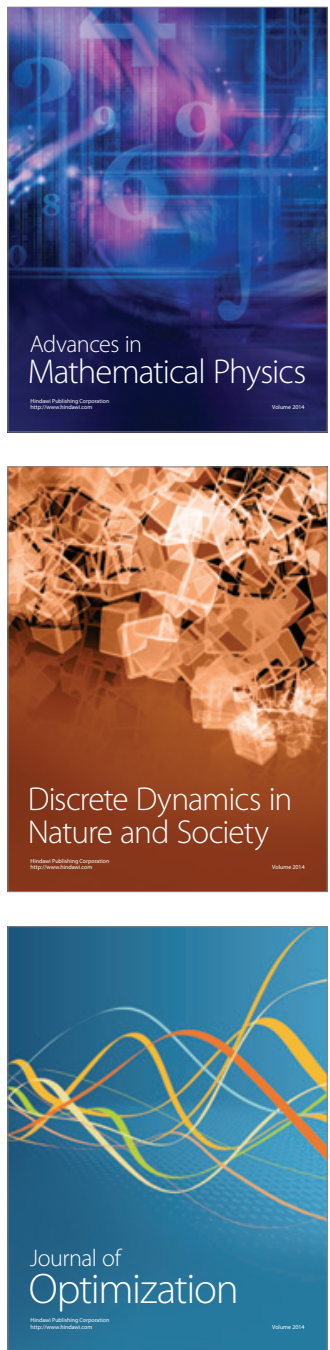